\title{
SUCULÊNCIA E SOLUBILIZAÇÃO DE PECTINAS DE MAÇÃS 'GALA' SUBMETIDAS A DIFERENTES TEMPOS DE RESFRIAMENTO E APLICAÇÃO DE 1-MCP ${ }^{1}$
}

\author{
Juiciness and pectins solubilization of 'Gala' apples submited to \\ different cooling times and 1-methylcyclopropene application
}

\author{
Rosangela Lunardi ${ }^{2}$, Auri Brackmann ${ }^{3}$, Anderson Machado de Mello, \\ Sérgio Tonetto de Freitas, ${ }^{5}$ Jocemar Francisco Zanatta ${ }^{6}$, Cesar Valmor Rombaldi
}

\begin{abstract}
RESUMO
Objetivou-se com este trabalho avaliar o efeito da aplicação pós-colheita de 1-metilciclopropeno (1-MCP, $625 \mathrm{~nL} \cdot \mathrm{L}^{-1}$ ) e tempo de resfriamento (Resfriamento Lento $(R L)=$ oito dias; Resfriamento Rápido $(R R)=$ três dias) em maçãs 'Gala' armazenadas por seis meses em armazenamento refrigerado (AR) sobre a perda de suculência, a firmeza de polpa, o conteúdo de pectina solúvel (PS) e a atividade das enzimas pectinametilesterase (PME) e poligalacturonase (PG). A temperatura de armazenamento foi de $0,5^{\circ} \mathrm{C}$. Ao final do período em $\mathrm{AR}$ e mais sete dias a $20^{\circ} \mathrm{C}$, foram avaliados a suculência, a firmeza de polpa, o conteúdo de PS e as atividades da PME e PG. A aplicação de 1-MCP manteve a firmeza mais elevada e não afetou a suculência das maçãs após seis meses em AR; o resfriamento rápido manteve as maçãs mais suculentas e firmes que o resfriamento lento após 7 dias a $20^{\circ} \mathrm{C}$.
\end{abstract}

Termos para indexação: Suculência, Malus domestica, qualidade.

\section{ABSTRACT}

The objective of this study was to evaluate the effect of postharvest application of 1-methylcyclopropene (1-MCP, 625 nL. $\mathrm{L}^{-1}$ ) and cooling time of 'Gala' apples (slow cooling (SC) for eight days or fast cooling (FC) for three days) on the juiciness loss, flesh firmness, soluble pectin content (SPC) and pectinmethylesterase (PME) and polygalacturonase (PG) activities after 6 months under refrigerated storage (RS). The storage temperature was $0.5^{\circ} \mathrm{C}$. At the end of the RS period, and more 7 days at $20^{\circ} \mathrm{C}$, the juiciness, flesh firmness, SPC, PME and PG were evaluated. Application of 1-MCP resulted in the highest firmness and it did not affect the juiciness of the apples after 6 months in RS; the fast cooling compared to slow cooling maintained apples juicier and firmer after 7 days at $20^{\circ} \mathrm{C}$.

Index terms: Juiciness, Malus domestica, quality.

(Recebido para publicação em 30 de junho de 2003 e aprovado em 22 de outubro de 2003)

\section{INTRODUÇÃO}

A 'Gala' é uma cultivar de maçã que foi rapidamente aceita na maior parte do mundo, por apresentar frutos com boa coloração e aparência, e polpa crocante e suculenta (DENARDI e CAMILO, 1992). Porém, essas características de crocância e suculência são rapidamente perdidas durante o armazenamento dos frutos.

Várias pesquisas têm sido conduzidas visando ao desenvolvimento de técnicas e tratamentos que pos- sam manter a qualidade dos frutos, ampliando a vida pós-colheita desses frutos. Entre essas técnicas, o 1metilciclopropeno (1-MCP), que é um composto volátil, vem sendo pesquisado para aplicação em pós-colheita de frutos, hortaliças e flores. Segundo Porat et al. (1999), o 1-MCP prende-se aos receptores celulares do etileno e inibe a ação do etileno endógeno e exógeno. Essa interação entre o 1-MCP e o sítio de ligação do etileno é irreversível, e os frutos tratados com 1-MCP eventualmente recuperam a capacidade de amadurecer,

\footnotetext{
1. Parte da tese desenvolvida pelo primeiro autor para obtenção do grau de Doutor em Ciências, Universidade Federal de Pelotas/UFPel - 96010-900 - Pelotas, RS.

2. Engenheira Agrônoma, Doutora em Ciências/UFPel.

3. Engenheiro Agrônomo, Dr. Professor do Departamento de Fitotecnia, Universidade Federal de Santa Maria/UFSM - 91119-900 - Santa Maria, RS

4. Engenheiro Agrônomo, aluno do curso de Pós-graduação em Agronomia/UFSM.

5. Aluno do Curso de Agronomia/UFSM.

6. Aluno do Curso de Agronomia/UFPel.

7. Engenheiro Agrônomo, Dr., Professor do Departamento de Ciência e Tecnologia Agroindustrial/UFPel.
} 
provavelmente pela formação de novos sítios de recepção do etileno (ARGENTA et al., 2001).

Muitos são os efeitos benéficos do 1-MCP em estender o período de vida pós-colheita e manter a qualidade de maçãs. O 1-MCP reduz a incidência de podridões (MIR e BEAUDRY, 2000) e distúrbios fisiológicos, como a escaldadura e o escurecimento interno da polpa (FAN et al., 1999b), reduz a taxa de respiração, produção de etileno, perda de firmeza de polpa e acidez titulável (FAN et al., 1999a).

Outra prática pós-colheita, que aliada à aplicação de 1-MCP, pode reduzir ainda mais as perdas póscolheita em armazenamento prolongado é o rápido resfriamento dos frutos. O resfriamento remove o calor de campo dos frutos, diminuindo o metabolismo respiratório (BROSNAN e SUN, 2001), retardando o amadurecimento (BRACKMANN et al., 2001). Segundo a maioria dos autores, o ideal seria resfriar e armazenar as maçãs imediatamente após a colheita. Nas grandes empresas, entretanto, é comum que o resfriamento demore de 8 a 10 dias, por causa das dificuldades inerentes à colheita, transporte e capacidade de refrigeração instalada.

Portanto, em razão da falta de informações sobre o comportamento da suculência em função do resfriamento e uso de 1-MCP, objetivou-se avaliar o efeito de diferentes períodos de resfriamento dos frutos e aplicação de 1-MCP sobre a qualidade de maçãs 'Gala' durante o armazenamento refrigerado.

\section{MATERIAL E MÉTODOS}

O experimento foi conduzido no Núcleo de Pesquisa em Pós-colheita (NPP) do Departamento de Fitotecnia da Universidade Federal de Santa Maria (UFSM) e no Laboratório de Biotecnologia do Departamento de Ciência e Tecnologia Agroindustrial da Universidade Federal de Pelotas (UFPel), durante o ano de 2002. Foram utilizadas maçãs 'Gala' procedentes de pomares comerciais da empresa Schio, de Vacaria, RS. No momento da colheita, a firmeza de polpa era de 74,5 N. Antes da instalação do experimento, os frutos foram selecionados, eliminando-se aqueles com lesões e baixo calibre, e homogeneizadas as amostras experimentais.

Os frutos foram acondicionados em armazenamento refrigerado $(\mathrm{AR})$ à temperatura de $0,5^{\circ} \mathrm{C}$ e umidade relativa do ar (UR) em torno de $95 \%$, durante seis meses. Esses frutos foram colocados em minicâmaras com volume de 232 litros, com o objetivo de manter alta a UR. O experimento foi um bifatorial com quatro tratamentos, dispostos no delineamento inteiramente casualizado com três repetições, sendo a unidade experimental composta por 20 frutos, para a determinação da firmeza de polpa, e 6 frutos para as demais determinações. Os tratamentos foram constituídos de dois períodos de resfriamento (Resfriamento Lento $(\mathrm{RL})=$ oito dias; Resfriamento Rápido $(\mathrm{RR})=$ três dias) associados com a aplicação de 625 nL.L L $^{-1}$ de 1-MCP. O tratamento-testemunha foi sem a aplicação de 1-MCP. O resfriamento dos frutos ocorreu de forma gradativa e contínua, partindo da temperatura de $25^{\circ} \mathrm{C}$ e chegando ao final do período de resfriamento com $0,5^{\circ} \mathrm{C}$. Isso foi feito com o abaixamento da temperatura da câmara de $6^{\circ} \mathrm{C}$ por dia durante três dias no RR e $2,5^{\circ} \mathrm{C}$ por dia durante oito dias no RL, para se obter a temperatura final de $0,5^{\circ} \mathrm{C}$. A temperatura foi monitorada com termômetros de mercúrio inseridos na polpa de frutos.

A aplicação de 1-MCP foi realizada no início do armazenamento, e como fonte de 1-MCP, utilizou-se o produto Agrofresh $(0,14 \%)$, na relação de $1,0 \mathrm{~g}$ de produto. $\mathrm{m}^{-3}$ para obter 625 nL.L $\mathrm{L}^{-1}$ de 1 -MCP na atmosfera da minicâmara. $\mathrm{O}$ produto foi solubilizado em $25 \mathrm{~mL}$ de água a $60^{\circ} \mathrm{C}$ em um recipiente hermético e, posteriormente, a solução foi transferida para uma placa de Petri, já no interior da minicâmara, que foi imediatamente fechada. Os frutos ficaram expostos ao produto durante 24 horas a $0,5^{\circ} \mathrm{C}$, e, em seguida, as minicâmaras foram ventiladas com o auxílio de uma bomba de sucção com vazão $14 \mathrm{~m}^{3} \cdot \mathrm{h}^{-1}$ durante uma hora.

Os frutos foram analisados após seis meses de armazenamento refrigerado e após sete dias de exposição a uma temperatura de $20^{\circ} \mathrm{C}$, com oscilação de $\pm 2^{\circ} \mathrm{C}$, simulando um período de comercialização. Os parâmetros analisados foram: firmeza de polpa, suculência, conteúdo de pectina solúvel e atividade das enzimas pectinametilesterase (PME) e poligalacturonase (PG). A firmeza de polpa (N) foi determinada com uso de penetrômetro manual, com ponteira de $11 \mathrm{~mm}$ de diâmetro. A suculência (\%) foi determinada por prensagem de $20 \mathrm{~g}$ de polpa durante um minuto, numa prensa pneumática especialmente desenvolvida para esse fim no NPP da UFSM. A quantidade de suco foi obtida pela diferença do peso inicial da amostra $(20 \mathrm{~g})$ e o peso final (após a prensagem). A suculência foi expressa em porcentagem, que representa gramas de suco por 100 gramas de polpa. Para as avaliações do conteúdo de pectina solúvel e da atividade das enzimas PME e PG, a polpa foi coletada e rapidamente congelada em nitrogênio líquido e mantida em $-20^{\circ} \mathrm{C}$ para posteriores análises. $\mathrm{O}$ conteúdo de pectina solúvel (mg de ácido 
galacturônico por $100 \mathrm{~g}$ de polpa) foi extraído segundo a técnica descrita por McCready e McComb (1952), sendo a determinação realizada colorimetricamente pela reação com carbazol, segundo Bitter e Muir (1962). A PME (U) foi determinada pela técnica empregada por Ratner et al. (1969). A unidade de atividade enzimática da pectinametilesterase foi considerada como sendo a quantidade de enzima capaz de catalisar a desmetilação de pectina correspondente a um nmol de $\mathrm{NaOH}$ por minuto nas condições do ensaio. A PG (U) foi extraída de acordo com Pressey e Avants (1973) e Jen e Robinson (1984). A determinação foi feita conforme a técnica de Somogyi, modificada por Nelson (1944). A unidade de atividade enzimática da $\mathrm{PG}$ foi definida como a quantidade de enzima capaz de catalisar a formação de um nmol de grupos redutores por minuto sob as condições do ensaio.

Os dados foram submetidos à análise de variância e as médias foram comparadas pelo teste de Duncan, a $5 \%$ de probabilidade. Os dados em porcentagem foram transformados pela fórmula arc.sen $\sqrt{x} / 100$, antes da análise de variância. Procedeu-se, também, a uma análise de correlação entre a firmeza de polpa e suculência com os frutos dos tratamentos com RL + 1-MCP e RR + 1-MCP analisados na saída da câmara. Utilizou-se uma unidade experimental de 20 frutos.

\section{RESULTADOS E DISCUSSÃO}

Observou-se que após seis meses de armazenamento refrigerado (AR), não houve diferença significativa na suculência das maçãs entre os tratamentos (Tabela 1). A firmeza de polpa desses frutos, no entanto, foi influenciada pela interação entre período de resfriamento e aplicação de 1-MCP, e a firmeza manteve-se mais elevada no tratamento com resfriamento rápido (RR) e aplicação de 1-MCP. Nos frutos sem a aplicação de 1-MCP, não houve diferença estatística entre o resfriamento lento $(R L)$ e RR. Já naqueles tratados com 1-MCP, a firmeza foi maior no RR, possivelmente porque nos frutos nãotratados a firmeza estava baixa demais para que se observasse alguma diferença entre os tratamentos, ao passo que, nos tratados, o 1-MCP realçou essa diferença. O efeito do 1-MCP ficou evidente, pois tanto no RL como no RR, os frutos tratados com 1-MCP mantiveram a firmeza de polpa mais elevada. No en- tanto, esse efeito benéfico do 1-MCP só foi observado na firmeza das maçãs; para a porcentagem de suculência, não houve diferença significativa entre os tratamentos (Tabela 1).

Quando os frutos foram analisados após sete dias a $20^{\circ} \mathrm{C}$, observou-se que o tratamento com RR apresentou frutos com maior suculência e firmeza de polpa (Tabela 1). A tendência esperada era de que frutos com maior firmeza de polpa apresentassem maior suculência, como foi constatado no tratamento com RR. A aplicação de 1-MCP, por sua vez, determinou frutos com maior firmeza, embora não tenha influenciado sua suculência. A falta de suculência é uma característica de fruto com polpa farinhenta.

Pre-Aymard et al. (2002) observaram que a aplicação de 1-MCP preveniu o desenvolvimento de polpa farinhenta em maçãs 'Anna', que é uma cultivar precoce e que perde rapidamente a firmeza e a suculência, à semelhança da maçã 'Gala'. Pelos resultados observados na Tabela 1 , os tratamentos com 1-MCP e com RR, a exemplo do observado na saída da câmara, mantiveram os frutos mais firmes, embora não houvesse interação dos tratamentos (Tabela 1). Os benéficos efeitos da RR na manutenção da firmeza de polpa concordam com os encontrados por Brackmann et al. (2000, 2001). Brosnan e Sun (2001) acreditam que o RR seja a chave para o sucesso do armazenamento de frutos e hortaliças, pois o RR diminui o metabolismo e retarda a maturação (BRACKMANN e BALZ, 2000).

Os efeitos do 1-MCP em manter a firmeza de polpa são observados para diversos frutos, como abacate (FENG et al., 2000; JEONG et al., 2002), abricó (FAN et al., 2000), ameixa (ARGENTA et al., 2002a) quivi e caqui (ARGENTA et al., 2002b), morango (JIANG et al., 2001) e especialmente maçã (FAN et al., 1999b; FAN e MATTHEIS, 1999; ARGENTA et al., 2000; RUPASINGHE et al., 2000; ARGENTA et al., 2001; FAN e MATTHEIS, 2001; DEELL et al., 2002; PRE-AYMARD et al., 2002). Na literatura, poucos trabalhos puderam ser encontrados sobre o efeito do 1-MCP em pêssegos, porém, Argenta et al. (2002a) observaram, após 10 dias de prateleira, que os frutos tratados com 1-MCP apresentaram firmeza de polpa semelhante ou superior aos frutos não tratados, dependendo do estádio de maturação na colheita. No entanto, o 1-MCP causou aumento da severidade de dano de frio. 
TABELA 1 - Firmeza de polpa e suculência de maçãs 'Gala' após 6 meses de armazenamento refrigerado e após mais 7 dias de exposição a $20^{\circ} \mathrm{C}$, submetidas a diferentes períodos de resfriamento e concentrações de 1-MCP. Santa Maria, 2003.

\begin{tabular}{|c|c|c|c|c|c|c|c|}
\hline \multirow{2}{*}{$\begin{array}{c}\text { Data de } \\
\text { Avaliação }\end{array}$} & \multirow{2}{*}{$\begin{array}{c}\text { Concentr. de } \\
\text { 1-MCP } \\
\left(\text { nL.L. } \mathbf{L}^{-1}\right)\end{array}$} & \multicolumn{3}{|c|}{$\begin{array}{l}\text { Firmeza de polpa } \\
(\mathrm{N})\end{array}$} & \multicolumn{3}{|c|}{$\begin{array}{l}\text { Suculência } \\
(\%)\end{array}$} \\
\hline & & RL & $\mathbf{R R}$ & Média & RL & $\mathbf{R R}$ & Média \\
\hline \multirow{4}{*}{$\begin{array}{l}\text { Saída da } \\
\text { câmara }\end{array}$} & 625 & 53,7 a $B^{*}$ & 65,4 a A & - & 79,2 & 77,8 & $78,5 \mathrm{a}$ \\
\hline & 0 & $50,1 \mathrm{~b} \mathrm{~A}$ & 49,4 b A & - & 77,7 & 75,7 & $76,7 \mathrm{a}$ \\
\hline & Média & - & - & & $78,5 \mathrm{~A}$ & $76,8 \mathrm{~A}$ & \\
\hline & $\mathrm{CV}(\%)$ & & 3,48 & & & 1,97 & \\
\hline \multirow{4}{*}{$\begin{array}{c}\text { Sete dias a } \\
20^{\circ} \mathrm{C}\end{array}$} & 625 & 55,2 & 62,5 & 58,8 a & 77,6 & 80,4 & $79,0 \mathrm{a}$ \\
\hline & 0 & 48,4 & 49,7 & $49,1 \mathrm{~b}$ & 76,2 & 80,7 & $78,4 \mathrm{a}$ \\
\hline & Média & $51,8 \mathrm{~B}$ & $56,1 \mathrm{~A}$ & & $76,9 \mathrm{~B}$ & $80,6 \mathrm{~A}$ & \\
\hline & $\mathrm{CV}(\%)$ & & 4,33 & & & 2,82 & \\
\hline
\end{tabular}

*Médias seguidas de mesma letra na coluna e na linha não diferem entre si pelo teste de Duncan (5\%).

A solubilização da pectina e a atividade das enzimas PG e PME não foram bons parâmetros para explicar o comportamento da suculência, tanto quando os frutos foram analisados na saída da câmara, como após sete dias a $20^{\circ} \mathrm{C}$, pois apresentaram resultados contraditórios entre si. Nos frutos analisados na saída da câmara, o conteúdo de pectina solúvel foi maior nos tratamentos com resfriamento lento (RL) e sem a aplicação de 1-MCP (Tabela 2), evidenciando que poderia ter havido maior atividade das enzimas $\mathrm{PME}$ e PG, que são as principais enzimas que despolimerizam e solubilizam pectinas. No entanto, não foi nesses frutos que houve a maior atividade da PME e PG, pelo contrário, a atividade da PG foi maior nos frutos com 1-MCP e RR, sem diferença na PME (Tabela 2). Conforme Awad (1993), o fenômeno normal que ocorre durante o amadurecimento dos frutos é o aumento da solubilização de pectinas e aumento da atividade da PG, enquanto a PME aumenta, diminuiu ou permanece constante, de- pendendo do fruto. Feng et al. (2000), por sua vez, observando a atividade da PG em abacate tratados com 1MCP, verificaram que nesses frutos a PG teve menor atividade, ao passo que os frutos não tratados apresentaram maior atividade. Por esse resultado, observa-se que o 1-MCP foi eficaz em retardar o amadurecimento, pois reteve a atividade da PG. No entanto, os resultados do presente trabalho contrariam o obtido por esses autores, talvez porque essa enzima seja sensível à rápida diminuição da temperatura, conforme o observado no tratamento com RR, pois foi nesse tratamento que a enzima atingiu a maior atividade. Fato esse que não aconteceu após sete dias dos frutos a $20^{\circ} \mathrm{C}$ (Tabela 2), em que apesar de não ter havido diferença estatística da atividade da PG entre os tratamentos, verificou-se que os frutos submetidos ao RL apresentaram maior atividade, confirmado pelos resultados de pectina solúvel, que foi maior nesses frutos (Tabela 2). 


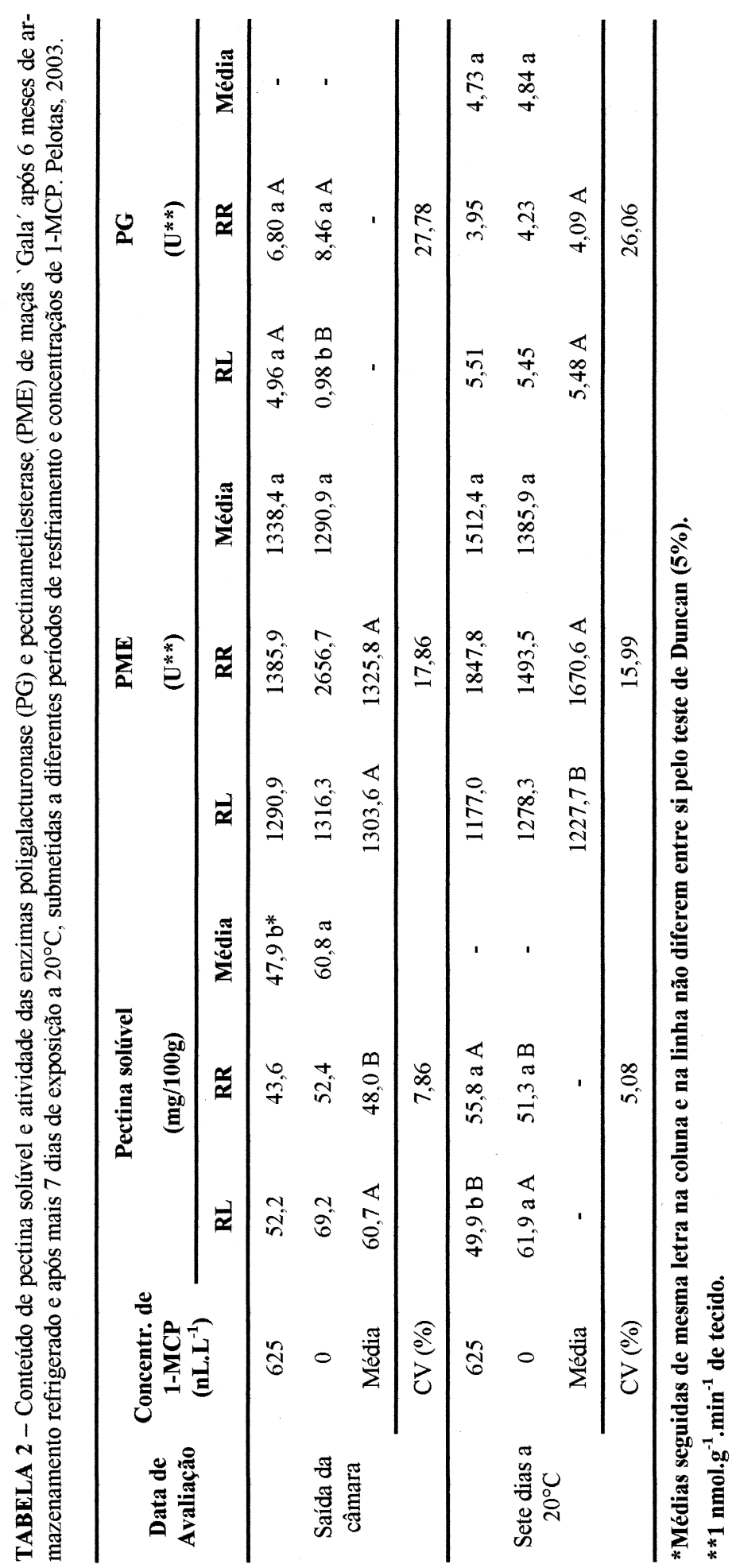

Ciênc. agrotec., Lavras, v. 28, n. 4, p. 871-877, jul./ago., 2004 
Conforme observado na Tabela 2, houve interação entre os períodos de resfriamento e a aplicação de 1-MCP, para a pectina solúvel, e a maior solubilização da pectina ocorreu nos frutos submetidos ao RL sem aplicação de 1-MCP. Quanto ao efeito do 1-MCP, Jeong et al. (2002) observaram que o conteúdo de pectina solúvel em frutos que não foram tratados com 1-MCP aumentou significativamente até o amadurecimento total do fruto. Já naqueles tratados com 1-MCP, esses autores verificaram que houve um atraso na solubilização da pectina, pois, no início do amadurecimento, ocorreu um decréscimo nos níveis de pectina solúvel, porém, no decorrer do amadurecimento, esses níveis aumentaram, atingindo os mesmos níveis dos frutos-controle, quando amadureceram totalmente.

A atividade da PME, por sua vez, foi menor no tratamento com RL, sem diferir no tratamento com 1MCP (Tabela 2). Por outro lado, Jeong et al. (2002) observaram que frutos tratados com 1-MCP apresentaram menor atividade da PME que frutos não-tratados. Nestes, a PME declinou para níveis muito baixos em frutos totalmente maduros. No entanto, para esses autores, apesar de o 1-MCP retardar o amadurecimento, a tendência é de que os frutos tratados com 1-MCP atinjam os mesmos níveis de PME quando estiverem completamente maduros, quando comparados aos frutoscontrole no mesmo estádio de maturação.

\section{CONCLUSÕES}

A aplicação de 1-MCP não tem efeito na manutenção da suculência de maçãs 'Gala' armazenadas por seis meses em armazenamento refrigerado, porém, mantém a firmeza de polpa mais elevada.

$\mathrm{O}$ resfriamento rápido em até três dias mantém as maçãs mais suculentas e mais firmes após sete dias de exposição a $20^{\circ} \mathrm{C}$.

O conteúdo de pectina solúvel e atividade das enzimas pectinametilesterase e poligalacturonase não afetam a suculência de maçãs 'Gala'.

\section{REFERÊNCIAS BIBLIOGRÁFICAS}

ARGENTA, L. C.; FAN, X.; MATTHEIS, J. P. Efeitos do tratamento 1-MCP e atmosfera controlada sobre a conservação da qualidade de maçãs 'Gala', 'Fuji' e 'Braeburn'. In: ENCONTRO NACIONAL DE FRUTICULTURA DE CLIMA TEMPERADO ENFRUTE, 4., 2001, Fraiburgo. Anais... Caçador: EPAGRI, 2001. p. 165-169.
ARGENTA, L. C.; FAN, X.; MATTHEIS, J.; KRAMMES, J. G.; MEGGUER, C. Possibilidades de aumento da conservação pós-colheita de frutas de caroço pelo uso de 1-MCP. In: ENCONTRO NACIONAL DE FRUTICULTURA DE CLIMA TEMPERADO ENFRUTE, 5., 2002, Fraiburgo. Anais... Caçador: EPAGRI, 2002a. p. 265-269.

ARGENTA, L. C.; KRAMMES, J. G.; MEGGUER, C.; VIEIRA, M.; CRESTÂNI, F. Possibilidades de aumento da conservação da qualidade após a colheita de caqui e quivi pelo uso de 1-MCP. In: ENCONTRO NACIONAL DE FRUTICULTURA DE CLIMA TEMPERADO - ENFRUTE, 5., 2002, Fraiburgo. Anais... Caçador: EPAGRI, 2002b. p. 270-274.

ARGENTA, L. C.; MATTHEIS, J.; FAN, X. Impacto do 1-MCP (1-metilciclopropeno) sobre a conservação da qualidade de maçãs e pêras. In: ENCONTRO NACIONAL DE FRUTICULTURA DE CLIMA TEMPERADO - ENFRUTE, 3., 2000, Fraiburgo. Anais... Caçador: EPAGRI, 2000. p. 129-133.

AWAD, M. Fisiologia pós-colheita de frutos. São Paulo: Nobel, 1993. 114 p.

BITTER, T.; MUIR, H. M. A modified uronic acid carbazole reaction. Analytical Chemistry, New York, v. 34, p. 330-334, 1962.

BRACKMANN, A.; BALZ, G. Efeito do préresfriamento e tratamentos químicos pós-colheita sobre a qualidade da maçã 'Gala' após o armazenamento em atmosfera controlada. Revista Científica Rural, Bagé, v. 5, n. 2, p. 12-20, 2000.

BRACKMANN, A.; HUNSCHE, M.; LUNARDI, R. Efeito do atraso no início do armazenamento e do tempo de pré-resfriamento sobre a qualidade da maçã 'Gala'. Ciência e Agrotecnologia, Lavras, v. 25, n. 1, p. 143-148, 2001.

BRACKMANN, A.; HUNSCHE, M.; STEFFENS, C. A. Qualidade da maçã 'Gala' (Malus domestica Borkh.) submetida a diferentes períodos de préresfriamento dos frutos e concentrações de etileno. Revista Brasileira de Fruticultura, Jaboticabal, v. 22, p. 1-5, 2000. Edição Especial.

BROSNAN, T.; SUN, D. W. Precooling techniques and applications for horticultural products: a review. International Journal of Refrigeration, Amsterdam, v. 24, p. 154-170, 2001. 
DEELL, J. R.; MURR, D. P.; PORTEOUS, M. D. Influence of temperature and duration of 1methylcyclopropene (1-MCP) treatment on apple quality. Postharvest Biology and Technology, Amsterdam, v. 24, n. 3, p. 349-353, 2002.

DENARDI, F.; CAMILO, A. P. Cultivares de macieira para o Sul do Brasil. Hortisul, Pelotas, v. 2, n. 3, p. 1219, 1992.

FAN, X.; ARGENTA, L. C.; MATTHEIS, J. P. Inhibition of ethylene action by 1-methylcyclopropene prolongs storage life of apricots. Postharvest Biology and Technology, Amsterdam, v. 20, n. 2, p. 135-142, 2000.

FAN, X.; BLANKENSHIP, S. M.; MATTHEIS, J. P. 1Methylcyclopropene inhibits apples ripening. Journal of the American Society Horticultural Science, Alexandria, v. 124, n. 6, p. 690-695, 1999a.

FAN, X.; MATTHEIS, J. P. 1-methylcyclopropene and storage temperature influence responses of 'Gala' apple fruit to gamma irradiation. Postharvest Biology and Technology, Amsterdam, v. 23, n. 2, p. 143-151, 2001.

FAN, X.; MATTHEIS, J. P. Impact of 1methylcyclopropene and methyl jasmonate on apple volatile production. Journal of Agricultural and Food Chemistry, Washington, v. 47, n. 7, p. 2847-2853, 1999.

FAN, X.; MATTHEIS, J. P.; BLANKENSHIP, S. M. Development of apple superficial scald, soft scald, core flush, and greasiness is reduced by MCP. Journal of Agricultural and Food Chemistry, Washington, v. 47, n. 8, p. 3063-306. 1999 b.

FENG, X.; APELBAUM, A.; SISLER, E. C.; GOREN, $R$. Control of ethylene responses in avocado fruit with 1-methylcyclopropene. Postharvest Biology and Technology, Amsterdam, v. 20, n. 2, p. 143-150, 2000.

JEN, J. J.; ROBINSON, M. L. P. Pectolytic enzymes in sweet bell peppers (Capsicum annuum L.). Journal of Food Science, Chicago, v. 49, n. 4, p. 1085-1087, 1984.

JEONG, J.; HUBER, D. J.; SARGENT, S. A. Influence of 1-methylcyclopropene (1-MCP) on ripening and cell wall matrix polygalacturonase of avocado (Persea americana) fruit. Postharvest Biology and Technology, Amsterdam, v. 25, n. 3, p. 241-256, 2002.
JIANG, Y.; JOYCE, D. C.; TERRY, L. A. 1methylcyclopropene treatment affects strawberry fruit decay. Postharvest Biology and Technology, Amsterdam, v. 23, n. 3, p. 227-232, 2001.

McCREADY, R. M.; McCOOMB, E. A. Extraction and determination of total pectic materials in fruits. Analytical Chemistry, Washington, v. 24, n. 12, p. 1586-1588, 1952.

MIR, N. A.; BEAUDRY, R. M. Use of 1-MCP to reduce the requirement for refrigeration and modified-atmospheres in the storage of apple fruit. In: INTERNATIONAL CONFERENCE ON POSTHARVEST SCIENCE, 2000, Jerusalem. Abstracts... Jerusalem: ISHS/ARO, 2000. p. 23.

NELSON, N. A. A photometric adaptation of Somogyi method for the determination of glucose. Journal of Biological Chemistry, Baltimore, v. 135, n. 1, p. 136175, 1944.

PORAT, R.; WEISS, B.; COHEN, L.; DAUS, A.; GOREN, R.; DROBY, S. Effects of ethylene and 1methylcyclopropene on the postharvest qualities of 'Shamouti' oranges. Postharvest Biology and Technology, Amsterdam, v. 15, n. 2, p. 155-163, 1999.

PRE-AYMARD, C.; WEKSLER, A.; LURIE, S. Responses of 'Anna', a rapidly ripening summer apple, to 1-methylcyclopropene. Postharvest Biology and Technology, Amsterdam. No prelo.

PRESSEY, R.; AVANTS, J. K. Separation and characterization of the exopolygalacturonase and endopolygalacturonase from peaches. Plant Physiology, Baltimore, v. 52, n. 3, p. 252-256, 1973.

RATNER, A. R.; GOREN, R.; MONSELISE, S. Activity of pectin esterase and cellulase in the abscission zone of citrus leaf explants. Plant Physiology, Washington, v. 44, n. 12, p. 1717-1723, 1969.

RUPASINGHE, H. P. V.; MURR, D. P.; PALIYATY, G.; SKOG, L. Inhibitory effect of 1$\mathrm{MCP}$ on ripening and superficial scald development in 'McIntosh' and 'Delicious' apples. Journal Horticultural Science and Biotechnology, Ashford, v. 75, n. 3, p. 271-276, 2000. 